\author{
Ana María Morales Guijarro ${ }^{1, *}$ \\ Pilar Arribas Sancho² \\ Raquel Díaz Díaz ${ }^{3}$ \\ David Guadarrama Ortega ${ }^{4}$
}

1. Enfermera de hospitalización de medicina Interna. Corresponsable de la implantación de la Guía de buenas prácticas: valoración del riesgo y prevención de úlceras por presión. Vocal de la comisión de heridas cutáneas crónicas. Vocal de la comisión de calidad. Hospital Universitario Fundación Alcorcón. Madrid. España.

2. Supervisora de la Unidad de Hospitalización de Medicina Interna. Responsable de la implantación de la Guía de buenas prácticas: valoración del riesgo y prevención de úlceras por presión. Presidenta de la comisión de heridas cutáneas crónicas. Vocal de la comisión de calidad. Hospital Universitario Fundación Alcorcón. Madrid. España.

3. Enfermera de hospitalización medicina interna. Impulsora en la implantación de la Guía de buenas prácticas: valoración del riesgo y prevención de úlceras por presión. Vocal de la comisión de seguridad del paciente. Hospital Universitario Fundación Alcorcón. Madrid. España.

4. Enfermero. Investigación Innovación y Desarrollo. Unidad de Investigación. Hospital Universitario Fundación Alcorcón. Madrid. España.

*Autor para correspondencia.

Correo electrónico: anaguijarro@msn.com (Ana María Morales Guijarro).

Recibido el 17 de junio de 2020; aceptado el 26 de enero de 2021.

\section{Impacto de la implantación de la guía de buenas prácticas: valoración del riesgo y prevención de lesiones por presión en ámbito hospitalario} Impact of the implementation of the guide of good practices: risk assessment and prevention of pressure ulcers in hospital settings

\section{RESUMEN}

Objetivo: Evaluar la implantación de la guía de buenas prácticas (GBP): Valoración del riesgo y prevención de lesiones por presión (LPP) en el ámbito hospitalario, en la unidad de medicina interna del Hospital Universitario Fundación Alcorcón (HUFA) de la Comunidad de Madrid. Metodología: Estudio cuasiexperimental pre-post donde se evalúan los indicadores de evaluación antes (2017) y después (2019) de la implantación de la GBP. Variables: LPP intrahospitalarias, valoración y revaloración del riesgo de LPP, aplicación de superficies de control de presión en pacientes con riesgo de LPP, momento de desarrollo de LPP y categorías de lesiones desarrolladas. Resultados: La muestra analizada fue de 764 pacientes. La incidencia de LPP en 2017 fue del 2,70\%; en 2018, del 6,1\%, y en 2019, del 4,1\%. La valoración del riesgo de LPP en 2017 fue de $94,59 \%$ frente a un $98,57 \%$ en 2019 . La revaloración del riesgo pasó de un $0 \%$ antes de la implantación de la guía a un 94,3\% tras su implantación. La aplicación de superficies especiales de manejo de presión en 2017 fue del 7,69\%, y a finales de 2019 ascendió a un 70,67\%.

Conclusiones: La implantación de la GBP produjo una disminución de la incidencia en el desarrollo de LPP durante el ingreso, un aumento significativo en la revaloración de los pacientes con riesgo de LPP y en la utilización de superficies especiales de manejo de presión.

PALABRAS CLAVE: Úlceras por presión, guía de práctica clínica, prevención, enfermería, riesgo, incidencia.

\section{ABSTRACT}

Objective: To evaluate the implementation of the good practice guide: risk assessment and prevention of pressure ulcers in a hospital environment, in the internal medicine unit of the University Hospital Fundación Alcorcón of the Community of Madrid. Methodology: a quasi-experimental, pre-post study in which indicators are evaluated before (2017) and after (2019) the implementation of the good practice guide. Variables: incidence rate of pressure injuries, assessment and reassessment of the risk of pressure injuries, application of pressure control surfaces in patients at risk of pressure injuries, time of development of pressure injuries, and categories of injuries developed. Results: the analyzed sample consists of 764 patients. The incidence rate of pressure injuries in 2017 was a 2.70\%; a 6,1\% in 2018 and a 4,1\% in 2019. The risk assessment of pressure injuries in 2017 was $98,57 \%$, compared to $94,3 \%$ in 2019 . Regarding the reassessment, it goes from a $0 \%$ before the implementation a $96.49 \%$ after. The application of special surfaces for handling pressure in 2017 was a 7.69\%, and a 70,67\% at the end of 2019. Conclusions: a decrease of the incidence rate in the development of pressure injuries during the patients' admission, a significant increase in the reassessment of the patients at risk of pressure injuries and in the use of special surfaces for handling pressure.

KEYWORDS: Pressure injuries, clinical practice guide, prevention, nursing, risk, incidence rate.

\section{У INTRODUCCIÓN}

La organización mundial de la salud (OMS), en su informe sobre la salud en el mundo del año 2010, destaca la promoción y la protección de la salud como aspectos esenciales para el bienestar humano y para lograr un desarrollo socioeconómico sostenido ${ }^{1}$.
Las guías de buenas prácticas (GBP) son documentos basados en la evidencia científica sobre temas clínicos y entornos laborales saludables, que incluyen recomendaciones específicas para enfermeras, profesionales de la salud, pacientes, familiares y cuidadores informales. Del mismo modo, aportan recomendaciones específicas para docentes, responsables de formación y/o calidad y gestores. La Asociación Profesional de Enfer- 
meras de Ontario (RNAO, por sus siglas en inglés) hace hincapié en que la prevención eficaz de las lesiones por presión (LPP) requiere la labor de un equipo multidisciplinar. La misión de la guía consiste en ayudar a las enfermeras a proporcionar unos cuidados basados en la evidencia a aquellos adultos con riesgo de padecer LPP. Las enfermeras desempeñan un papel fundamental en la valoración del riesgo y su prevención ${ }^{2}$. El proceso de implementación tiene como propósito primordial lograr que los usuarios finales, prestadores y pacientes utilicen las recomendaciones planteadas por las guías de la práctica clínica (GPC) en la práctica clínica diaria ${ }^{3}$.

Los cuidados basados en la evidencia aumentan la calidad de los cuidados, la seguridad en los pacientes y el manejo de los riesgos en la práctica; además, permitirán dilucidar cuáles son los enfoques más exitosos y de mejor costo/efectividad en los cuidados de enfermería, y así establecer la mejor condición posible para ofrecer un excelente cuidado a los pacientes ${ }^{4,5}$.

Las LPP son un problema de salud mundial que limita la vida de los pacientes y sus familiares, y su prevención es un indicador de calidad en el ámbito de la salud ${ }^{2,6}$. En la actualidad, el interés en el cuidado relacionado con estas lesiones ha ido en aumento, no solo encaminado hacia la consecución de un tratamiento eficaz, sino también dirigido hacia la prevención, ya que se estima que hasta el $95-98 \%$ de las LPP son evitables 6

Según la RNAO, un 10\% de las personas que ingresan en un hospital desarrollan una LPP. Aproximadamente, el 60\% de los problemas relacionados con LPP se desarrollan en unidades de cuidados de pacientes agudos, normalmente en las dos primeras semanas de hospitalización ${ }^{2}$.

Las LPP y su tratamiento repercuten en la calidad de vida del paciente y de su familia, además de suponer elevados costes a los que las sufren y al sistema sanitario. Una intervención temprana es esencial para las personas con riesgo de presentar $\mathrm{LPP}^{2}$. En España, el costo total del tratamiento de las LPP en un ańo es aproximadamente de 461 millones de euros, cerca del 5\% del gasto sanitario anual. De este montante, el $15 \%$ representa el costo de apósitos y otros materiales, mientras que el 19\% representa el costo del tiempo de enfermería, y el $45 \%$ del total representa el costo de las estancias extra en el hospital relacionadas con estas lesiones ${ }^{7}$. En Reino Unido, los costos aumentan con el grado de úlcera porque el tiempo de curación es más largo y porque la incidencia de complicaciones es mayor en los casos más graves y suponen el $4 \%$ del gasto total sanitario. La mayor parte de este costo es tiempo de enfermería ${ }^{8}$.

El programa de implantación de buenas prácticas en cuidados surge en España del acuerdo entre tres instituciones comprometidas con la práctica basada en la evidencia: la Unidad de Investigación en Cuidados de Salud (Investén-isciii), el Centro Español para los Cuidados de salud Basados en la Evidencia (CECBE) y la Asociación de Enfermeras de Ontario (RNAO). El objetivo de esta acción es fomentar, facilitar y apoyar la implantación, evaluación y mantenimiento, en cualquier entorno de la práctica enfermera, de buenas prácticas en cuidados, basadas en las guías de la RNAO. Con ello se pretende crear una red nacional de centros comprometidos con la excelencia en cuidados que fidelice el uso de prácticas basadas en los mejores resultados de la investigación en cuidados?.

En España, la mayor fuente de estudios sobre prevalencia es la del GNEAUPP (Grupo Nacional para el Estudio y Asesoramiento en Úlceras por Presión y Heridas Crónicas). Disponer de datos acerca de la epidemiología de las LPP a nivel nacional aporta información de gran utilidad para poder hacer el dimensionamiento de este importante problema de salud y poder observar tendencias en su evolución. El último estudio, realizado en 2017, ha sido el primer estudio epidemiológico de ámbito nacional realizado en España que ha recogido datos sobre la prevalencia en unidades hospitalarias de los diferentes tipos de lesiones cutáneas relacionadas con la dependencia (LCRD) y no solo de las lesiones producidas por la presión. También es el estudio con una muestra más amplia y representativa de hospitales espańoles, sobre todo hospitales públicos, por lo que ofrece una información epidemiológica bastante fiable. La prevalencia de LCRD en unidades de hospitalización de adultos de hospitales públicos españoles fue, globalmente, del 8,7\%, siendo las LPP las lesiones más frecuentes, con un 7,0\% de prevalencia. Respecto a otros hospitales del mismo grupo (grupo 2: 200-499 camas), el estudio del GNEAUPP de 2017 muestra una prevalencia de LPP del 6,6\% ${ }^{10}$.

La prevalencia de LPP en España se mantiene en los últimos años por lo que, como avalan diversos estudios de investigación, la prevención es el método más eficiente para abordar el problema ${ }^{11,12}$. Así pues, la implantación de la guía de buenas prácticas es un paso fundamental para establecer unas recomendaciones conjuntas basadas en la evidencia científica.

Varios estudios indican dos puntos claves para la prevención de LPP: la importancia de un programa de educación del personal de enfermería $^{13,14}$-es fundamental realizar intervenciones educativas permanentes a los implicados en el cuidado preventivo de lesiones de piel para generar trasformaciones positivas en el equipo de salud y los cuidadores- ${ }^{15}$, y también se debe considerar de especial importancia la utilización de superficies especiales de manejo de presión (SEMP) como medida preventiva ${ }^{16}$.

El Hospital Universitario Fundación Alcorcón (HUFA) ha sido seleccionado como candidato a centro comprometido con la excelencia en cuidados (CECBE), en la 3. ${ }^{\text {a }}$ cohorte 2018-2020, dentro del programa de implantación de buenas prácticas en cuidados.

El municipio de Alcorcón tiene una superficie de $33,73 \mathrm{~km}^{2}$, cuenta según el padrón municipal para 2017 del INE con 168141 habitantes y una densidad de población de 4984,91 habitantes $/ \mathrm{km}^{2}$. El hospital de referencia para esta población es el HUFA, hospital público integrado en el grupo 2 de los hospitales públicos del Servicio Regional Madrileño de Salud (SERMAS).

La implantación de la guía se realizó, inicialmente, en la Unidad de Medicina Interna del HUFA, y posteriormente se extendió al resto de unidades de hospitalización, urgencias, cuidados críticos, reanimación, quirófano, atención primaria y centros sociosanitarios.

En la tabla 1 se citan algunas de las recomendaciones implantadas durante todo el proceso de la implantación en el HUFA.

\section{Y OBJETIVOS}

\section{Objetivo general}

Evaluar la implantación de la GBP: valoración del riesgo y prevención de LPP, en el ámbito hospitalario, en la unidad de medicina interna del HUFA de la Comunidad de Madrid.

\section{Objetivos específicos}

- Conocer la incidencia de LPP de categoría I, II, III y IV desarrolladas durante la estancia hospitalaria.

- Explorar el grado de utilización de superficies especiales de manejo de presión para prevenir las LPP.

- Conocer el registro realizado por parte del personal de enfermería de valoración y de revaloración del riesgo de LPP a través de una escala validada. 
Tabla 1. Resumen de algunas recomendaciones implantadas de la guía de buenas prácticas

\begin{tabular}{|c|c|c|}
\hline Valoración & $\begin{array}{l}1.1 \\
1.2 a \\
1.5 \\
1.6 b \\
1.7\end{array}$ & $\begin{array}{l}\text { Realizar una valoración íntegra de la piel al ingreso, y posteriormente todos los días a aquellos pacientes que tengan riesgo } \\
\text { de deterioro de la integridad cutánea } \\
\text { Valorar el riesgo de LPP a través de la escala de Norton/Braden } \\
\text { Desarrollar estrategias para la prevención LPP en cualquier entorno clínico } \\
\text { Identificar LPP, seguir recomendaciones de RNAO y valoración y manejo de LPP en las categorías del I al IV } \\
\text { Todos los datos se documentan en el momento de la valoración inicial y de la valoración continua }\end{array}$ \\
\hline Planificación & 2.1 & $\begin{array}{l}\text { Realizar un plan de cuidados individualizado, incluyendo paciente, familia y personal sanitario. El equipo utiliza los datos de } \\
\text { valoración continua combinada con el criterio clínico para identificar los factores de riesgo y recomendar un plan de cuidados }\end{array}$ \\
\hline Intervenciones & $\begin{array}{l}3.1 a \\
3.1 b \\
3.4 \\
3.5 \\
3.8 \\
3.9\end{array}$ & $\begin{array}{l}\text { Los pacientes con riesgo de desarrollar una LPP deben de utilizar colchón antiescaras } \\
\text { Si existe riesgo de LPP, establecer una programación de cambios posturales } \\
\text { Valorar, documentar y manejar de forma eficaz el dolor y establecer un plan de cuidados adecuado para prevención de LPP } \\
\text { Evitar el masaje sobre las prominencias óseas y zonas enrojecidas } \\
\text { Proteger la piel de una humedad excesiva y de la incontinencia para mantener la integridad de la piel } \\
\text { Valoración al ingreso y durante el período hospitalario de estado nutricional e hidratación }\end{array}$ \\
\hline Formación & $5.1 \mathrm{a}$ & Establecer programas de formación para la prevención dirigido a personal de forma periódica \\
\hline $\begin{array}{l}\text { Recomendaciones } \\
\text { para la organización }\end{array}$ & 6.3 & Respaldo institucional adecuado \\
\hline
\end{tabular}

\section{У MÉTODOS}

\section{Diseño}

Se realizó un estudio de intervención pre-post donde se evaluaron los indicadores del seguimiento del proyecto, antes de la implantación de la GBP de la RNAO (2017) y tras su implantación (2019).

La población de estudio fueron los pacientes ingresados en la Unidad de Medicina Interna del HUFA. Los periodos analizados fueron el último trimestre de 2017, el último semestre de 2018 y el primer semestre de 2019. La implantación de la guía se realizó de forma gradual en el último trimestre del 2018, considerándose el periodo pre- hasta finales del 2018 y el periodo post- desde principios del 2019.

Los datos de los pacientes se obtuvieron de la base de datos del proyecto "CarEvID+ Care Evaluation Indicators", plataforma para la evaluación de la implantación de buenas prácticas en cuidados en enfermería en instituciones sanitarias adheridas al Programa de Implantación de Guías de Buenas Prácticas en Cuidados a través de Centros Comprometidos con la Excelencia en Cuidados $\left(\mathrm{CCEC}^{\oplus}\right)$. Esta plataforma se alimenta mediante la introducción de datos por parte de los evaluadores, profesionales del HUFA que analizan la historia clínica electrónica para obtener los indicadores de cuidados definidos por el proyecto. De esta plataforma se obtienen posteriormente los informes mensuales, trimestrales o anuales con los resultados de la implantación de las recomendaciones.

Esta información ha sido recogida por evaluadores formados y entrenados, con una metodología común y según la guía de evaluación del programa CCEC ${ }^{\oplus}$.

Las variables analizadas, además de si el paciente corresponde al grupo pre- y postimplantación, fueron: edad, sexo, LPP, categoría máxima de la LPP, evaluación del riesgo de LPP, uso de SEMP en pacientes de riesgo.

La muestra de pacientes se obtuvo con base en los criterios que la RNAO utiliza para la evaluación de la implantación de la GBP. Concretamente se analizan los pacientes dados de alta durante los 5 últimos días hábiles de cada mes en la unidad de hospitalización del servicio de medicina interna.
La forma de medir el impacto fue mediante la comparación de los indicadores obtenidos de CarEvID+. Se comparó la incidencia de LPP antes y después de la implantación de la GBP. Otras variables estudiadas fueron la valoración y revaloración del riesgo de LPP a través de una escala validada, la prevención de lesiones a través de superficies de apoyo, y la categorización y frecuencia de LPP.

El análisis estadístico se realizó con el programa IBM SPSS Statistics for Windows, Version 21.0. Se realizó un análisis descriptivo de la muestra y se analizaron los grupos de pacientes preimplantación y postimplantación, para comprobar si eran homogéneos en cuanto a sexo y edad, usando el test de la $\chi^{2}$ y la $t$ de Student, respectivamente. Para el contraste de hipótesis entre variables cualitativas se utilizó el test de la $\chi^{2}$. Para el contraste entre variables continuas y cualitativas se utilizó la $t$ de Student. Para la comparación de la variable "Categoría máxima de la LPP” se utilizó el test de la U de Mann-Whitney. En todos los análisis se estableció el nivel de significación estadística con un valor de $p$ inferior a 0,05 .

Las estrategias desarrolladas para la implantación de la GBP están detalladas en la tabla 2.

Consideraciones éticas: esta investigación respeta la Ley Orgánica 3/2018, de 5 de diciembre, de Protección de Datos Personales y garantía de los derechos digitales. Los datos de los pacientes se analizaron de forma agregada y anonimizada mediante los informes generados automáticamente por la plataforma CarEvID+.

\section{Y RESULTADOS}

La muestra analizada fue de 764 pacientes, de los cuales 202 son anteriores a la implantación de la GBP y 562 posteriores a dicha implantación.

El 47,7\% de la muestra analizada fue de sexo femenino y el 52,3\% de sexo masculino, con una edad media de 76,2 años [desviación estándar (DE): 15,34 años].

El análisis de homogeneidad de los grupos estudiados en cuanto a la edad, sexo y riesgo de LPP al ingreso se muestra en la tabla 3.

El análisis del impacto de la GBP en los indicadores del proyecto BPSO se muestra en la tabla 4. 
Tabla 2. Estrategias para desarrollar la implantación de la guía de buenas prácticas

\begin{tabular}{|c|c|}
\hline $\begin{array}{l}\text { Basadas en recursos } \\
\text { humanos }\end{array}$ & $\begin{array}{l}\text { a. Formación de impulsores/evaluadores, corresponsable y responsable en la implantación de GBP } \\
\text { b. Reconocimientos dentro del equipo de trabajo de las figuras anteriormente descritas } \\
\text { c. Organización de actividades formativas institucionales (6 ediciones anuales de formación sobre LPP) } \\
\text { d. Reactivación de la comisión de heridas cutáneas crónicas } \\
\text { e. Dinámicas de equipo (desayunos saludables, brainstorming, etc.). } \\
\text { f. Creación de escuela de familia } \\
\text { g. Asistencia a congresos para actualización y revisión de conocimientos (enfermeras y TCAES) } \\
\text { h. Intercambio de experiencias con otros centros BPSO }{ }^{\circledR}\end{array}$ \\
\hline $\begin{array}{l}\text { Basadas en recursos } \\
\text { materiales }\end{array}$ & $\begin{array}{l}\text { a. Solicitud en el plan de inversión anual institucional de superficies especiales de manejo de presión (SEMP) } \\
\text { b. Aumento de dotación de sobrecolchones de presión alterna } \\
\text { c. Práctica reflexiva de uso adecuado de absorbentes (en proceso el estudio de investigación por parte del equipo) } \\
\text { d. Remodelación de infraestructuras de la unidad para favorecer deambulación precoz y segura } \\
\text { e. Diseño de póster informativos, trípticos, etc. } \\
\text { f. Diseño de reloj para control de cambios posturales }\end{array}$ \\
\hline
\end{tabular}

Tabla 3. Análisis de homogeneidad de los grupos en función de edad, sexo y riesgo de lesiones por presión al ingreso

\begin{tabular}{|l|c|c|c|c|}
\hline & $\begin{array}{c}\text { Pre-GBP } \\
(\mathbf{n = 2 0 2})\end{array}$ & $\begin{array}{c}\text { Post-GBP } \\
(\mathbf{n = 5 6 2 )}\end{array}$ & \multicolumn{1}{|c|}{ Test } & $\begin{array}{c}\text { Valor } \\
\text { de } \boldsymbol{p}\end{array}$ \\
\hline Edad media (DE) & $75,5(16,24)$ & $76,45(15,01)$ & $t$ de Student & 0,451 \\
\hline Sexo (hombres) \% (n) & $55,7 \%(111)$ & $51,1 \%(287)$ & $\chi^{2}$ & 0,258 \\
\hline $\begin{array}{l}\text { Riesgo de LPP al } \\
\text { ingres0 \% (n) }\end{array}$ & $28,2 \%(57)$ & $27,8 \%(155)$ & $\chi^{2}$ & 0,905 \\
\hline
\end{tabular}

DE: desviación estándar; GPB: guía de buenas prácticas; LPP: lesiones por presión.

Tabla 4. Análisis comparativo de los indicadores evaluados antes y después de la implantación de la guía de buenas prácticas

\begin{tabular}{|l|c|c|c|c|}
\hline & $\begin{array}{c}\text { Pre-GBP } \\
(\mathbf{n = 2 0 2})\end{array}$ & $\begin{array}{c}\text { Post-GBP } \\
(\mathbf{n = 5 6 2})\end{array}$ & $\begin{array}{c}\text { Test } \\
\text { Valor } \\
\text { de } \mathbf{p}\end{array}$ \\
\hline $\begin{array}{l}\text { Valoración del riesgo } \\
\text { de LPP al ingreso }\end{array}$ & $98 \%(198)$ & $98,6 \%(552)$ & 0,589 \\
\hline $\begin{array}{l}\text { Revaloración del } \\
\text { riesgo de LPP }\end{array}$ & $69,2 \%(137)$ & $94,3 \%(514)$ & & $<0,001$ \\
\hline $\begin{array}{l}\text { Uso de SEMP en } \\
\text { pacientes de riesgo }\end{array}$ & $19,3 \%(11 / 57)$ & $70,7 \%(106 / 150)$ & $\chi^{2}$ & $<0,001$ \\
\hline $\begin{array}{l}\text { Incidencia de LPP } \\
\text { intrahospitalaria }\end{array}$ & $5,4 \%(119)$ & $4,1 \%(23)$ & & 0,43 \\
\hline
\end{tabular}

GPB: guía de buenas prácticas; LPP: lesiones por presión; SEMP: superficies especiales de manejo de presión.

Tabla 5. Análisis completo de la variable categoría máxima de la lesión por presión (LPP)

\begin{tabular}{|c|c|c|c|c|}
\hline $\begin{array}{c}\text { Categoría máxima } \\
\text { de la LPP }\end{array}$ & Pre $(n=24)$ & Post $(n=60)$ & Test & $\begin{array}{l}\text { Valo } \\
\text { de } p\end{array}$ \\
\hline Cat. I & $29,2 \%(7)$ & $15 \%(9)$ & \multirow{5}{*}{$\begin{array}{l}\text { U de Mann- } \\
\text { Whitney }\end{array}$} & \multirow{5}{*}{0,203} \\
\hline Cat. II & $50 \%(12)$ & $50 \%(30)$ & & \\
\hline Cat. III & $8,3 \%(2)$ & $20 \%(12)$ & & \\
\hline Cat. IV & $12,5 \%(3)$ & $6,7 \%(4)$ & & \\
\hline Cat. V & $0,00 \%$ & $8,3 \%(5)$ & & \\
\hline
\end{tabular}

La categoría máxima de la LPP más frecuente en ambos grupos fue la categoría II, ambas con un 50\% de los casos. El análisis completo de la variable categoría máxima de la LPP se muestra en la tabla 5.

La evolución anual de la incidencia de LPP durante el ingreso se muestra en la figura 1: pasó del 2,70\% en 2017 al 5,4\% en 2018 y al $3,93 \%$ en 2019.

Tras la evaluación de los resultados se observa un aumento en el indicador de revaloración del riesgo de lesiones por presión, pasando del $0 \%$ en el año 2017 al 96,3\% en 2019 ( $p<0,001)$. La valoración del riesgo de LPP al ingreso es realizada en un $94,59 \%$ en 2017 y un $98,6 \%$ en 2019 $(p<0,162)$. En el año 2017 se aplicaron al 7,7\%,y en 2019 al 70,7\% $(p<0,001)$; todo ello se refleja en la figura 2.

Con respecto a la aparición de LPP se observa que la categoría II es la que aparece en mayor porcentaje durante todo el periodo estudiado, aunque se observa una tendencia descendente, tal como se muestra en la figura 3 .

\section{У DISCUSIÓN}

Se observa un aumento en la incidencia de LPP $(6,1 \%)$ en 2018 con respecto a la medición basal (2,70\%) en 2017 que atribuimos a la concienciación de los profesionales de la importancia del registro en la historia clínica electrónica. Tras la aplicación de las recomendaciones de la guía se observa un descenso $(4,1 \%)$ en 2019.

Cabe destacar que antes de la implantación de la guía existía en el hospital una política oficial en cuanto al registro de la valoración del riesgo de LPP que se realiza en las primeras 24 horas de la estancia hospitalaria, pero no en cuanto la realización de la revaloración, que era llevada a cabo por el personal de enfermería según sus criterios individuales. Esto se modificó tras la implantación y se convirtió también en política oficial del hospital, realizándose diariamente a todos los pacientes con riesgo de LPP en el turno de mañana. También hay que tener en cuenta el posible sesgo de infrarregistro existente antes de la implantación de la guía $\mathrm{BPSO}^{\oplus}$.

Según un estudio publicado en 2014 por Sebastián-Viana et al., el número de pacientes que presentan LPP prácticamente se duplica si la recogida de datos es observacional frente al registro en la historia clínica $^{18}$. Esto podría aplicarse, en nuestro caso, a los datos referidos en 2017. En este mismo estudio se declaró, en aquel hospital, una prevalencia del 4,2\%. 


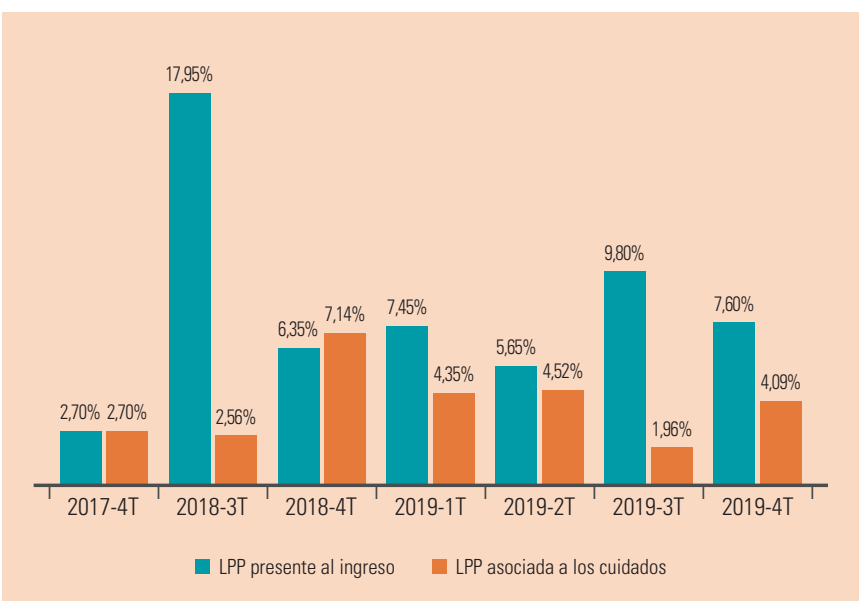

Figura 1. Incidencia de lesiones por presión en el Hospital Universitario Fundación Alcorcón.

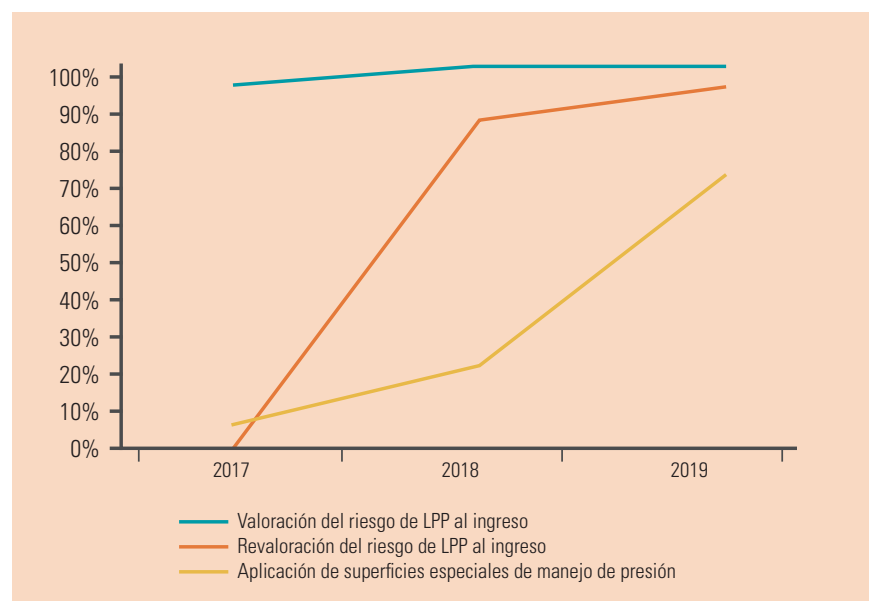

Figura 2. Registro de valoración y revaloración del riesgo de lesiones por presión a través de una escala validada. Registro de aplicación de superficies especiales de manejo de presión.

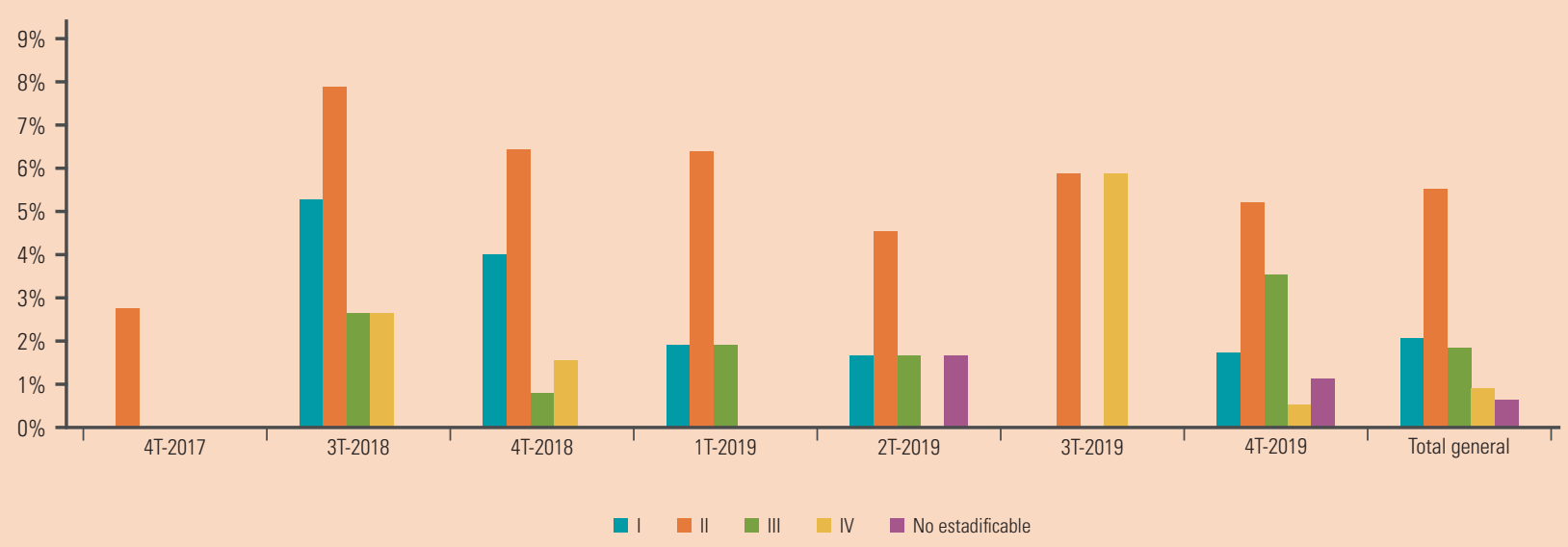

Figura 3. Incidencias de lesiones por presión, según su categoría, desarrolladas durante la estancia hospitalaria.

Frente a los datos publicados de LPP, en 2004, los hospitales de Ontario declararon que uno de cada 4 pacientes desarrolló una LPP durante el ingreso; la incidencia en nuestro estudio ha cambiado tras la implantación de la GBP. A nivel europeo, en un estudio de prevalencia de LPP realizado en 2006, en el que participaron 5047 pacientes, se obtuvo que un $18,1 \%$ tenía una o más LPP ${ }^{19}$. En Colombia, la incidencia de LPP oscila entre el $7,7 \%$ y $26,9 \%{ }^{20}$.

Se necesitarían estudios a nivel nacional de incidencia para poder comparar nuestro estudio, ya que la mayor parte de los estudios encontrados miden la prevalencia de LPP.

Si observamos la clasificación de LPP según su categoría se observa que la categoría II es la más frecuente, dato que coincide con los estudios de prevalencia nacionales que se han realizado en los últimos años en España ${ }^{10-12}$.

En cuanto a la utilización de SEMP cabe destacar que el esfuerzo realizado por el equipo de implantación en la formación de los profesionales, unido al aumento de la dotación de recursos materiales por parte de la dirección del hospital, facilitó su utilización en todas las unidades del hospital. Diversos estudios indican una frecuencia en la aplicación de SEMP entre el $29,7 \%$ y el $44 \%{ }^{21,22}$, mientras que en nuestro estudio se observa un aumento hasta el $72 \%$ tras la implantación de la GBP.
Una de las limitaciones de nuestro estudio ha sido el infrarregistro antes de la implantación de la GBP, lo que puede no reflejar en su totalidad la realidad en la práctica clínica. Algunos estudios destacan la importancia de las intervenciones frecuentes y repetidas para mantener los éxitos de mejora de la calidad, en lo que se refiere a la documentación de las intervenciones realizadas ${ }^{23}$.

\section{$У$ CONCLUSIONES}

Los datos provisionales de la incorporación de la GBP de valoración del riesgo y prevención de las úlceras por presión en la Unidad de Medicina Interna del HUFA nos permiten concluir que:

- Tras la implantación completa de la GBP se observa una disminución de la incidencia en el desarrollo de lesiones por presión durante la estancia hospitalaria.

- Aumento significativo en la revaloración de los pacientes con riesgo de LPP.

- Aumento significativo en la utilización de SEMP. 
Basándonos en los primeros resultados obtenidos en este estudio, se observa en los profesionales de enfermería un aumento de la sensibilización respecto a la prevención y valoración de úlceras por presión tras la implementación de GBP.

Todo ello supone una mejora en los resultados en salud para los pacientes, con impacto en los cuidadores. Aunque no se ha realizado el estudio económico, sería necesario su análisis para conocer el impacto de la implantación de la guía en los recursos sanitarios del sistema

\section{Conflicto de intereses}

Los autores manifiestan que no tienen ningún conflicto de intereses relacionado con este estudio.

\section{Agradecimientos}

A la Dirección de Operaciones y Enfermería (DOE) por el apoyo institucional y a todos los profesionales de enfermería que trabajan en la implantación de la Guía de Buenas Prácticas.

\section{У BIBLIOGRAFÍA}

1. Organización Mundial de la Salud. Informe sobre la salud en el mundo: la financiación de los sistemas de salud: el camino hacia la cobertura universal. [Internet]. Ginebra: Organización Mundial de la Salud; 2010 [consultado 10 de marzo de 2020]. Disponible en: https://apps.who.int/iris/handle/10665/44373

2. RNAO-INVESTEN. Guía de buenas prácticas en enfermería: cómo enfocar el futuro de la enfermería. Valoración del riesgo y prevención de las úlceras por presión. [Internet]. Ontario: RNAO; 2005 [consultado 10 de marzo de 2020]. Disponible en: https:// evidenciaencuidados.es/bpso/recursos/

3. Duarte A, Torres A, Vélez C. Manual de implementación de guías de práctica clínica basadas en evidencia en instituciones prestadoras de servicios de salud en Colombia. Bogotá: Ministerio de Salud y Protección social; 2014

4. Urra-Medina E, Retamal-Valenzuela C, Tapia-Pinto CF, Rodríguez-Vidal M. Enfermería basada en la evidencia: qué es, sus características y dilemas. Investig Educ Enferm. 2010;28(1): $108-18$

5. Bajnok I, Grinspun D, Lloyd M, McConnell H. Liderando mejoras de calidad mediante el desarrollo, implementación, y medición de las guías de buenas prácticas de Enfermería. MedUNAB 2015 [consultado 15 de marzo de 2020);17(3):155-62. Disponible en: https://revistas.unab.edu.co/index.php/medunab/article/view/2382

6. Avilés Martinez MJ, Sánchez Lorente M. Guía de práctica clínica para el cuidado de personas con úlceras por presión o riesgo de padecerlas. Valencia: Generalitat Valenciana. Conselleria de Sanitat; 2012 [consultado 15 de marzo de 2020) Disponible en: https://portal.quiasalud.es/wp-content/uploads/2018/12/ GPC_520_Ulceras_por_presion_compl.pdf

7. Soldevilla Agreda JJ, Torra i Bou JE, Posnett J, Verdú Soriano J, San Miguel L, Mayan Santos JM. Una aproximación al impacto del coste económico del tratamiento de las úlceras por presión en España. Gerokomos. 2007;18(4):201-10. doi:10.4321/S1134 928X2007000400007

8. Bennet G, Dealey CPJ. The cost of pressure ulcers in the UK. Age Ageing. 2004;33(3):230-5. doi:10.1093/ageing/afh086

9. Centro español para los cuidados de salud basados en la evidencia [Internet]. Madrid: CECBE: 2013 [consultado 20 de marzo de 2020]. Disponible en: https://www.evidenciaencuidados.es/ es/index.php/implantacion/11-implantacion/41-proyecto-implantacion

10. Pancorbo-Hidalgo PL, García-Fernández FP, Pérez-López C Soldevilla Agreda JJ. Prevalencia de lesiones por presión y otras lesiones cutáneas relacionadas con la dependencia en población adulta en hospitales españoles: resultados del $5^{0}$ Estudio Nacional de 2017. Gerokomos. 2019;30(2):76-86

11. Pancorbo-Hidalgo PL, García-Fernández FP, Torra i Bou JE, Verdú Soriano J Soldevilla-Agreda JJ. Enidemiología de las úlceras por presión en España en 2013: $4^{\circ}$ Estudio Nacional de Prevalencia. Gerokomos. 2014;25(4):162-70.

12. Soldevilla Agreda JJ, Torra i Bou J Verdú Soriano JLCP $3^{\text {er Estu- }}$ dio Nacional de Prevalencia de Úlceras por Presión en España, 2009: Epidemiología y variables definitorias de las lesiones y pacientes. Gerokomos. 2011;22(2):77-90.

13. Sinclair L, Berwiczonek H, Thurston N, Butler S, Bulloch G, Ellery C GG. Evaluation of an evidence-based education program for pressure ulcer prevention. J Wound Ostomy Continence Nurs. 2004;31(1):43-50

14. Gunningberg L. Pressure ulcer prevention: evaluation of an education programme for Swedish nurses. J Wound Care. 2004:13(3):85-9.
15. González-Consuegra RV Pérez-Valderrama DC, Valbuena-Flor LF Prevención de lesiones de piel: educación en el equipo de salud y familiares de personas hospitalizadas. Rev. Fac. Med. 2016:64(2):229-38.

16. Peris Armero AG-CV. Prevención de úlceras por presión en el paciente encamado: nuevas evidencias. RECIEN. 2018;16:36-58.

17. IBM Corp. Released 2012. IBM SPSS Statistics for Windows, Version 21.0. Armonk, NY: IBM Corp.

18. Sebastián-Viana T, González-Ruiz JM, Núñez-Crespo F, Lema-Lorenzo I, Gadea-Romero G, Losa-Iglesias ME. La validez de un registro clínico de úlceras por presión. An Sist Sanit Navar. 2014;37(1). doi: 10.4321/S1137/66272014000100003

19. Vanderwee K, Clark M, Dealey C, Gunningberg L. Pressure ulcer prevalence in Europe: a pilot study. J Eval Clin Pr. 2007;13(2):227-35. doi:10.1111 / j.1365-2753.2006.00684. x.

20. González-Consuegra RV, Cardona-Mazo DM, Murcia-Trujillo PA, Matiz-Vera G. Prevalencia de úlceras por presión en Colombia: Informe preliminar. Rev Fac Med. 2014:62(3):369-77. doi:10.15446/revfacmed.v62n3.43004

21. Chaboyer W, Bucknall T, Gilles B, Thalib L, McInnes E. Adherence to evidence-based pressure injury prevention guidelines in routine clinical practise: A longitudinal study. Int Wound J. 2017;14:1290-8. doi:10.1111/iwj.81279

22. Gunninberg I, Stotts N, Idvall E. Hospital-acquired pressure ulcers in two Swedish Country Council: Cross-sectorial data as the doundation for future quality imporvement. Int Wount $J$ 2011:467-73. doi:10.1111/j1742-481x.2011.00818. x.

23. Dahlstrom $J$, et al. Improving identification and documentation or pressure ulcers al an urban academic hospital. J Comm J Qual Patient Saf. 2011;37:123-30. doi:10.1016/s15537250(11)37015-8 